\title{
Article
}

\section{Silica-bonded propylpiperazine- $N$-sulfamic acid as recyclable solid acid catalyst for preparation of 2-amino-3-cyano-4-aryl-5,10-dioxo- 5,10-dihydro-4H-benzo[g]chromenes and hydroxy-substituted naphthalene-1,4-dione derivatives}

\author{
Fahime Khorami, Hamid Reza Shaterian* \\ Department of Chemistry, Faculty of Sciences, University of Sistan and Baluchestan, PO Box 98135-674, Zahedan, Iran
}

\section{A R T I C L E I N F O}

\section{Article history:}

Received 31 August 2013

Accepted 4 November 2013

Published 20 February 2014

\section{Keywords:}

Silica-bonded propylpiperazine- $N$ -

sulfamicacid

Aldehyde

Malononitrile

Amine

Solvent-free

2-Hydroxynaphthalene-1,4-dione

\begin{abstract}
A B S T R A C T
An efficient method for the synthesis of 2-amino-3-cyano-4-aryl-5,10-dioxo-5,10-dihydro-4H-benzo[g]chromenes and hydroxy-substituted naphthalene-1,4-dione derivatives, using silica-bonded propylpiperazine- $N$-sulfamic acid as a solid acid, green, heterogeneous catalyst, under ambient and solvent-free conditions, is described. A simple procedure, high yields, short reaction time, safety, and reusability of the catalyst are advantages of these protocols.
\end{abstract}

(C) 2014, Dalian Institute of Chemical Physics, Chinese Academy of Sciences. Published by Elsevier B.V. All rights reserved.

\section{Introduction}

Multicomponent reactions (MCRs) are valuable tools in organic and medicinal chemistry [1]. The use of MCRs has become a major driving force in the development of highly efficient and sustainable procedures for the assembly of biologically active compounds, in both academia and industry [1,2].

2-Amino-3-cyano-4-aryl-5,10-dioxo-5,10-dihydro-4H-benzo $[g]$ chromene derivatives show various biological activity, including anticancer [3], anti-inflammatory [4], antimalarial [5,6], and pesticide activity [7]. Hydroxy-substituted naphthalene-1,4-dione derivatives are fluorescent heterocyclic compounds. These fluorescent materials are of interest in many disciplines and are used as emitters in electroluminescence devices [8], molecular probes for biochemical research [9], in traditional textile and polymer fields [10], and as fluorescent whitening agents [11].

A literature survey showed that 2-amino-3-cyano-4-aryl5,10-dioxo-5,10-dihydro-4H-benzo $[\mathrm{g}$ ] chromenes have been prepared in the presence of $\mathrm{DBU}\left[\mathrm{CH}_{3} \mathrm{COO}\right]$, [Pyrr] $\left[\mathrm{CH}_{3} \mathrm{COO}\right]$, [Pyrr][HCOO], [Pip][CH $\left.{ }_{3} \mathrm{COO}\right],[\mathrm{Pip}][\mathrm{HCOO}],[\mathrm{Hmim}][\mathrm{HCOO}]$, and 3-HPAA [12], $\mathrm{Et}_{3} \mathrm{~N}$ and $\mathrm{CH}_{3} \mathrm{CN}$ [13], $\mathrm{HOAc}$ /dimethylformamide [14], [bmim] $\mathrm{OH}$ [15], triethylbenzylammonium chloride [16], DBU [17], $\mathrm{Et}_{3} \mathrm{~N}$ and EtOH [18], 2-hydroxyethylammonium formate and 2-hydroxyethylammonium acetate [19], and cetyltrimethylammonium bromide [20] as catalysts from three-component condensation reactions of aromatic aldehydes, malononitrile, and 2-hydroxynaphthalene-

\footnotetext{
* Corresponding author. Tel: +98-541-2447010; Fax: +98-541-2431067; E-mail: hrshaterian@chem.usb.ac.ir DOI: 10.1016/S1872-2067(12)60761-X | http://www.sciencedirect.com/science/journal/18722067 | Chin. J. Catal., Vol. 35, No. 2, February 2014
} 
1,4-dione. Also, hydroxy-substituted naphthalene-1,4-dione derivatives have been synthesized in the presence of

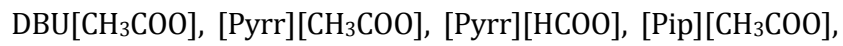
[Pip][HCOO], [Hmim][HCOO], and 3-HPAA [12], $\mathrm{InCl}_{3}$ [21], and nano copper(II) oxide [22] as catalysts in the three-component condensation reactions of aromatic aldehydes, aromatic amines, and 2-hydroxynaphthalene-1,4-dione.

In a continuation of our studies on the use of solid acid catalysts in organic transformations [23-27], we report an efficient method for the synthesis of 2-amino-3-cyano-4-aryl-5,10dioxo-5,10-dihydro-4H-benzo[ $g$ ]chromenes, using silicabonded propylpiperazine- $N$-sulfamic acid (SBPPSA) [28-31] as the catalyst, by three-component condensation reactions of aromatic aldehydes, malononitrile, and 2-hydroxynaphthalene1,4-dione under ambient and solvent-free conditions (Scheme 1).

We also used this environmentally friendly SBPPSA in the condensation reactions of aromatic aldehydes, aromatic amines, and 2-hydroxynaphthalene-1,4-dione in the preparation of hydroxy-substituted naphthalene-1,4-dione derivatives under ambient and solvent-free conditions (Scheme 2).

\section{Experimental}

All reagents were purchased from Merck or Aldrich and used without further purification. SBPPSA was prepared by the reaction of 3-piperazine- $N$-propylsilica with chlorosulfonic acid in chloroform, according to the method reported in the literature [28]. All yields refer to isolated products after purification. Nuclear magnetic resonance (NMR) spectra were recorded using a Bruker Advance DPX $300 \mathrm{MHz}$ instrument. The spectra were measured in DMSO- $d_{6}$ relative to tetramethylsilane. Infrared (IR) spectra were recorded using a JASCO FTIR 460 Plus spectrophotometer. Melting points were determined in open capillaries using a BUCHI 510 melting point apparatus. Thinlayer chromatography (TLC) was performed on silica-gel Poly Gram SIL G/UV 254 plates.

2.1. General procedure for synthesis of 2-amino-3-cyano-4aryl-5,10-dioxo-5,10-dihydro-4H-benzo[g]chromenes under

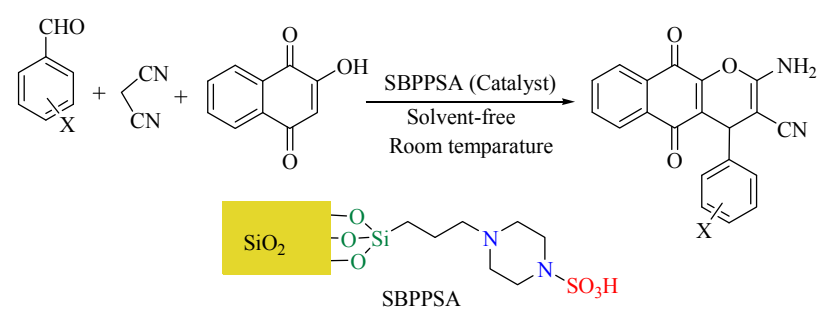

Scheme 1. Synthesis of 2-amino-3-cyano-4-aryl-5,10-dioxo-5,10dihydro- $4 H$-benzo $[g]$ chromene derivatives.

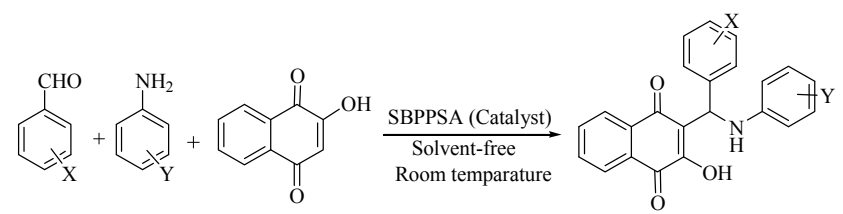

Scheme 2. Synthesis of hydroxy-substituted naphthalene-1,4-dione derivatives. solvent-free and ambient conditions

A mixture of an aldehyde (10 mmol), malononitrile (10 mmol), 2-hydroxynaphthalene-1,4-dione (10 mmol), and SBPPSA (0.05 g; 0.25 g equals $0.31 \mathrm{mmol}$ of $\mathrm{H}^{+}$[28]) as a solid acid catalyst was stirred under ambient and solvent-free conditions for a specific time. Completion of the reaction was monitored using TLC. Ethanol ( $5 \mathrm{~mL}$ ) was then added, the reaction mixture was filtered, and the remaining solid was washed with warm ethanol $(3 \times 5 \mathrm{~mL})$ to separate the catalyst. The filtrate solution containing the crude product was evaporated and recrystallized from ethanol. The recovered catalyst was dried and reused in subsequent runs. All of the desired product(s) were characterized by comparison of their physical data with those of known compounds.

Selected spectra for a known product are given below. 2-Amino-3-cyano-4-(2,4-dichlorophenyl)-5,10-dioxo-5,10-dihy dro- $4 H$-benzo $[g]$ chromene (Table 2, entry 13): orange powder; m.p. $=288{ }^{\circ} \mathrm{C}$; IR $\left(\mathrm{KBr}, \mathrm{cm}^{-1}\right): v_{\max } 3467,3341,3168,2201$, 1664, 1631, 1591, 1364, 1247, 1200; ${ }^{1} \mathrm{H}$ NMR (300 MHz, DMSO-d6): $\delta 4.74(1 \mathrm{H}, \mathrm{s}, \mathrm{CH}), 8.08-7.48\left(9 \mathrm{H}, \mathrm{m}, \mathrm{Ar}\right.$ and $\mathrm{NH}_{2}$ ); ${ }^{13} \mathrm{C}$ NMR (75 MHz, DMSO- $d_{6}$ ): $\delta 37.1,56.9,110.3,119.2,119.5$, 121.1,126.2 (2C), 126.5, 129.3, 131.2, 131.3, 133.1, 136.6 (2C), 136.9,149.5, 158.1, 177.2, 183.6.

\subsection{Synthesis of hydroxy-substituted naphthalene-1,4-dione derivatives under ambient and solvent-free conditions}

A mixture of an aldehyde (10 mmol), aniline $(10 \mathrm{mmol})$, 2-hydroxynaphthalene-1,4-dione (10 mmol), and SBPPSA (0.05 g; 0.25 g equals $0.31 \mathrm{mmol}$ of $\mathrm{H}^{+}$[28]) as a solid acid catalyst was stirred under ambient and solvent-free conditions for a specific time. Completion of the reaction was monitored using TLC. Ethanol ( $5 \mathrm{~mL}$ ) was added, the reaction mixture was filtered, and the remaining solid was washed with warm ethanol $(3 \times 5 \mathrm{~mL})$ to separate the catalyst. The filtrate solution containing the crude product was evaporated and recrystallized from ethanol. The recovered catalyst was dried and reused in subsequent runs. All of the desired product(s) were characterized by comparison of their physical data with those of known compounds.

Selected spectra for a known product are given below. 2-Hydroxy-3-[phenyl(phenylamino)methyl]naphthalene-1,4-di one (Table 3, entry 1): orange powder; m.p. $=145-146{ }^{\circ} \mathrm{C}$; IR $\left(\mathrm{KBr}, \mathrm{cm}^{-1}\right): v_{\max } 3434,3321,1660,1636 ;{ }^{1} \mathrm{H}$ NMR $(300 \mathrm{MHz}$, DMSO- $\left.d_{6}\right): \delta 5.98(1 \mathrm{H}, \mathrm{s}, \mathrm{CH}), 7.25-8.07(15 \mathrm{H}, \mathrm{m}, \mathrm{Ar}$ and $\mathrm{NH})$, $9.26(1 \mathrm{H}, \mathrm{s}, \mathrm{OH}) ;{ }^{13} \mathrm{C}$ NMR (75 MHz, DMSO- $\left.d_{6}\right): \delta 37.3,113.4$, 123.9, 125.9, 125.9, 126.4, 128.5, 129.6, 130.3, 132.6, 133.1, $133.5,135.0,138.1,146.0,156.8,157.6,181.8,184.0$.

\section{Results and discussion}

First, the reaction conditions were optimized using the reaction of 4-methoxybenzaldehyde, malononitrile, and 2-hydroxynaphthalene-1,4-dione in the presence of SBPPSA as a catalyst under ambient and solvent-free conditions as a model. The reaction was carried out with different amounts of SBPPSA 
Table 1

Optimization of amount of SBPPSA as catalyst in three-component synthesis of 2-amino-3-cyano-4-(4-methoxyphenyl)-5,10-dioxo-5,10dihydro- $4 H$-benzo $[g]$ chromene from the reaction of 2-hydroxynaphthalene-1,4-dione, malononitrile, and 4-methoxybenzaldehyde under solvent-free and ambient conditions.

\begin{tabular}{lccc}
\hline Entry & Catalyst $(\mathrm{g})$ & Time $(\mathrm{min})$ & Yield a $^{(\%)}$ \\
\hline 1 & 0.02 & 40 & 72 \\
2 & 0.05 & 25 & 90 \\
3 & 0.10 & 15 & 90 \\
4 & 0.20 & 9 & 91 \\
\hline
\end{tabular}

a Yields refer to pure isolated product.

(0.02, 0.05, 0.1, $0.2 \mathrm{~g}$; Table 1). As the data in Table 1 show, 0.05 $\mathrm{g}$ of SBPPSA afforded 2-amino-3-cyano-4-(4-methoxyphenyl)5,10 -dioxo-5,10-dihydro-4H-benzo $[g]$ chromene in $90 \%$ yield in $25 \mathrm{~min}$.

Next, the three-component condensation reactions of aro- matic aldehydes, malononitrile, and 2-hydroxynaphthalene1,4-dione for the preparation of 2-amino-3-cyano-4-aryl-5,10dioxo-5,10-dihydro- $4 H$-benzo $[g]$ chromene derivatives were investigated under the optimized conditions (Table 2). The reaction time and yields of the corresponding products were unaffected by the presence of electron-donating groups and electron-withdrawing groups on the benzaldehydes. We also examined aliphatic aldehydes such as $n$-heptanal instead of benzaldehydes in the reaction. All the starting materials were intact and none of the desired products or byproducts were formed after $24 \mathrm{~h}$ (Table 2, entry 16).

The proposed mechanism for the preparation of 2-amino3-cyano-4-phenyl-5,10-dioxo-5,10-dihydro-4H-benzo[g]chrom ene from benzaldehyde, malononitrile, and 2-hydroxynaphthalene-1,4-dione, using SBPPSA as a catalyst, is shown in Scheme 3. According to a literature report [13], 2-benzyli-

\section{Table 2}

Three-component synthesis of 2-amino-3-cyano-4-aryl-5,10-dioxo-5,10-dihydro-4H-benzo[g]chromene derivatives from reaction of 2-hydroxynaphthalene-1,4-dione, malononitrile, and aldehydes in the presence of SBPPSA as catalyst under solvent-free and ambient conditions.

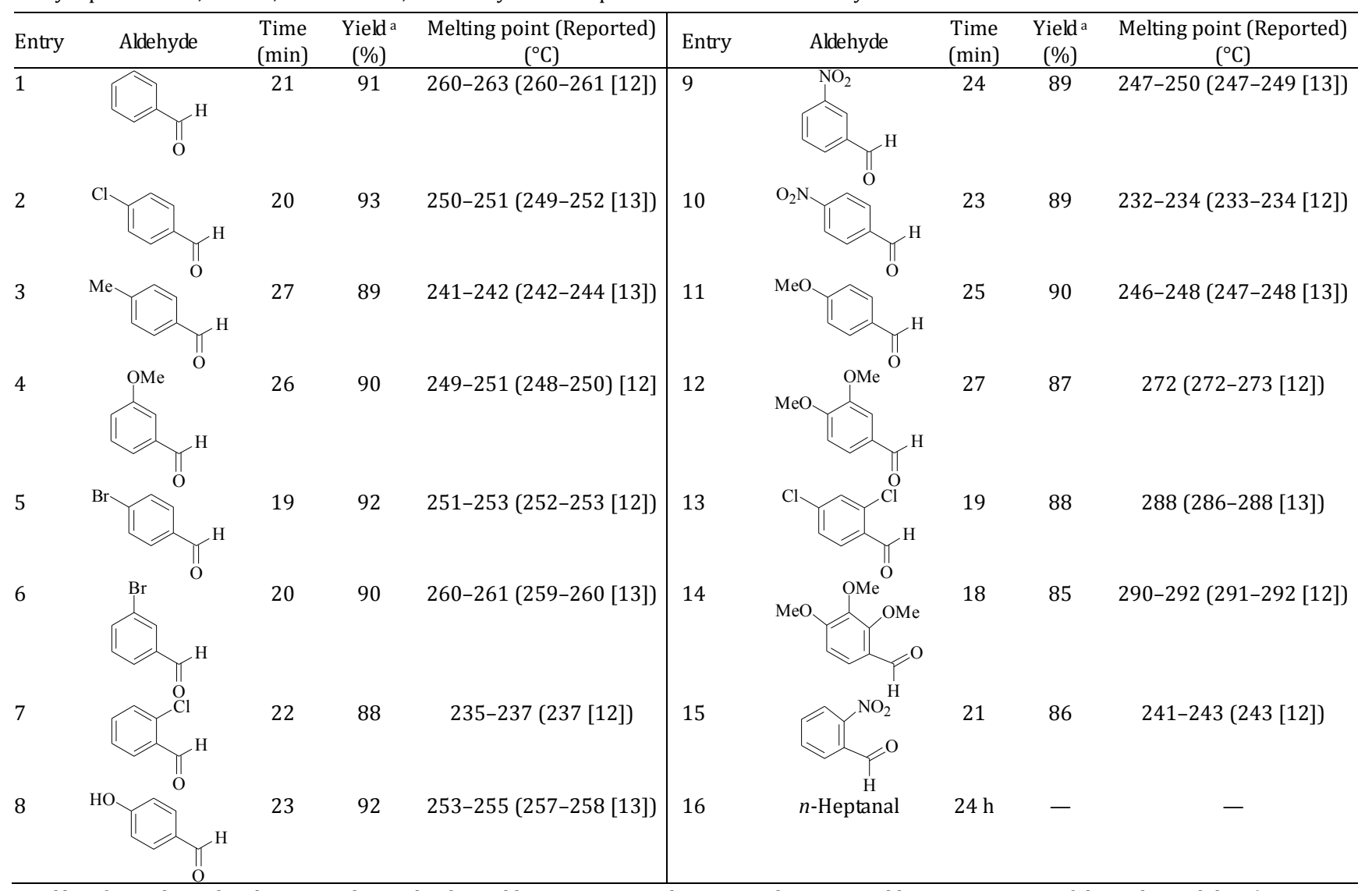

a Yields refer to the isolated pure products. The desired known pure products were characterized by a comparison of their physical data (m.p., IR, ${ }^{1} \mathrm{H}$ and ${ }^{13} \mathrm{C}$ NMR) with those of the known compounds.

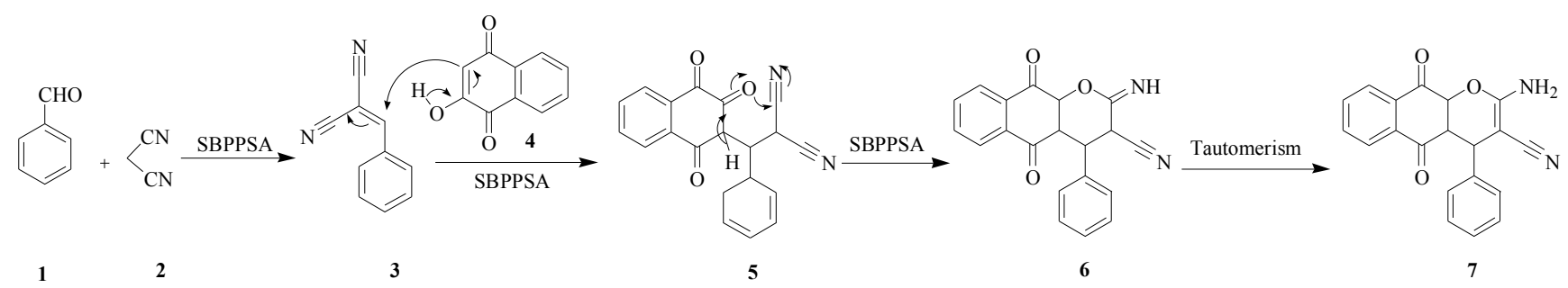

Scheme 3. Suggested mechanism for formation of 2-amino-3-cyano-4-phenyl-5,10-dioxo-5,10-dihydro-4H-benzo[ $\mathrm{g}] \mathrm{chromene}$. 


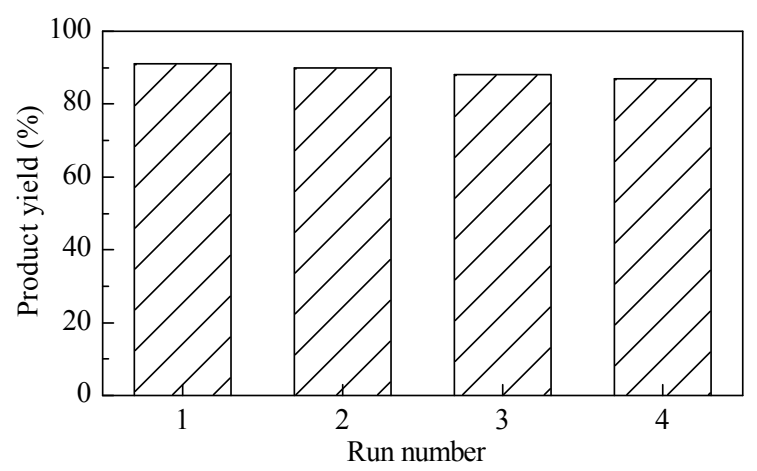

Fig. 1. Recycling of SBPPSA catalyst under ambient and solvent-free conditions for the reaction of benzaldehyde, malononitrile, and 2-hydroxynaphthalene-1,4-dione as a model.

denemalononitrile, which contains an electron-poor $\mathrm{C}=\mathrm{C}$ double bond, is formed by Knoevenagel addition of malononitrile to the benzaldehyde in the presence of an acid catalyst. The acid-activated 2-benzylidenemalononitrile is then attacked by 2-hydroxynaphthalene-1,4-dione (4), which leads to intermediate $\mathbf{5}$. Tautomerization and cyclization by nucleophilic attack of the $\mathrm{OH}$ group on the activated cyano $(\mathrm{CN})$ moiety gives intermediate 6. Subsequent tautomerization produces the desired product.

The possibility of recycling the catalyst was examined using the reaction of benzaldehyde, malononitrile, and 2-hydroxynaphthalene-1,4-dione for the preparation of 2-amino3-cyano-4-phenyl-5,10-dioxo-5,10-dihydro-4H-benzo[ $g]$ chrom ene, under the optimized conditions (Table 2, entry 1). After completion of the reaction, warm ethanol $(5 \mathrm{~mL})$ was added, the reaction mixture was filtered, and the remaining solid was washed with warm ethanol $(3 \times 5 \mathrm{~mL})$ to separate the catalyst. The recycled catalyst was used for the next reaction. The recycled catalyst could be reused at least four times without any appreciable loss of catalytic activity (Fig. 1).

The three-component condensation of aromatic aldehydes, anilines, and 2-hydroxynaphthalene-1,4-dione over $0.05 \mathrm{~g}$ of SBPPSA catalyst, under ambient and solvent-free conditions, for the preparation of hydroxy-substituted naphthalene-1,4diones was then investigated. A wide range of substituted and structurally diverse aldehydes and anilines was used, and the corresponding products were obtained in high to excellent yields (Table 3). $n$-Heptanal, an aliphatic aldehyde, produced only traces of the desired compounds and almost all of the starting materials were intact (Table 3 , entry 11 ).

The proposed mechanism for the preparation of 2-hydroxy3-[phenyl(phenylamino)methyl]naphthalene-1,4-dione from the reaction of benzaldehyde, aniline, and 2-hydroxynaphtha-
Table 3

Three-component synthesis of hydroxy-substituted naphthalene-1,4dione derivatives from reaction of 2-hydroxynaphthalene-1,4-dione, anilines, and aldehydes in the presence of SBPPSA as catalyst (Scheme 2) under solvent-free and ambient conditions.

\begin{tabular}{|c|c|c|c|c|c|}
\hline Entry & Aldehyde & Y & $\begin{array}{l}\text { Time } \\
(\min )\end{array}$ & $\begin{array}{l}\text { Yield }{ }^{a} \\
(\%)\end{array}$ & $\begin{array}{c}\text { Melting point (Reported) } \\
\left({ }^{\circ} \mathrm{C}\right)\end{array}$ \\
\hline 1 & & $\mathrm{H}$ & 8 & 91 & $145-146(144-146[12])$ \\
\hline 2 & $\mathrm{HO}$ & 4-Me & 11 & 91 & $150-152(151-152[12])$ \\
\hline 3 & & 4-Me & 9 & 90 & $142-145(143-144[21])$ \\
\hline 4 & $\mathrm{O}_{2} \mathrm{~N}$ & $\mathrm{H}$ & 9 & 87 & $145-147(145-148[21])$ \\
\hline 5 & & $\mathrm{H}$ & 8 & 89 & $184-186(189-190[12])$ \\
\hline 6 & $\mathrm{Me}$ & 4-Me & 10 & 91 & $145-149(144-147)[21]$ \\
\hline 7 & & $4-\mathrm{F}$ & 7 & 88 & $152-153(151[12])$ \\
\hline 8 & & 4-Me & 12 & 89 & 152 (151-152 [12]) \\
\hline 9 & & $4-\mathrm{F}$ & 9 & 88 & 151 (149-152 [21]) \\
\hline 10 & & 4-Me & 11 & 91 & $145-147$ (146 [12]) \\
\hline 11 & $n$-Heptanal & $4-\mathrm{F}$ & 480 & - & - \\
\hline
\end{tabular}

a Yields refer to the isolated pure products. The desired known pure products were characterized by a comparison of their physical data (m.p., IR, ${ }^{1} \mathrm{H}$ and ${ }^{13} \mathrm{C} \mathrm{NMR}$ ) with those of the known compounds.

lene-1,4-dione, using SBPPSA as a catalyst, is shown in Scheme 4. According to a literature report [21], an imine is formed by condensation of benzaldehyde and aniline. 2-Hydroxynaphthalene-1,4-dione is then added to the activated imine in the presence of an acid catalyst. Tautomerization converts intermediate $\mathbf{1 1}$ to the desired product.

Catalyst recycling was studied using a similar procedure to that described above, using the reaction of benzaldehyde, aniline, and 2-hydroxynaphthalene-1,4-dione as a model. The re-

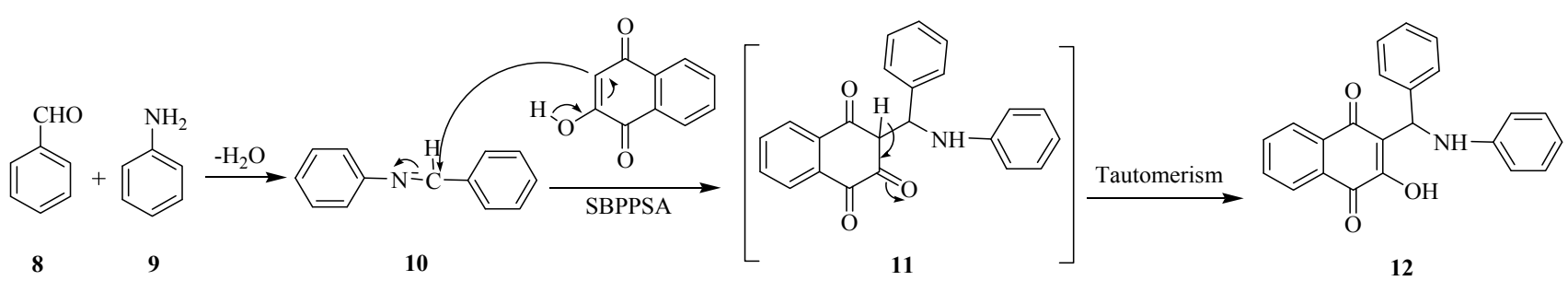

Scheme 4. Suggested mechanism for formation of 2-hydroxy-3-[phenyl(phenylamino)methyl]naphthalene-1,4-dione. 


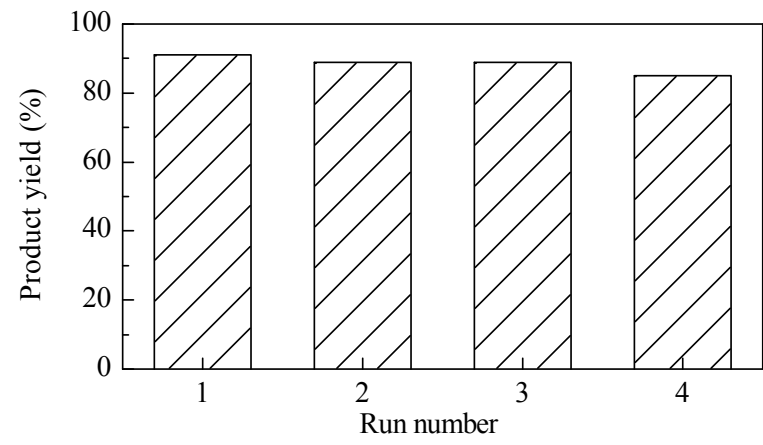

Fig. 2. Recycling of SBPPSA catalyst under ambient and solvent-free conditions for the reaction of benzaldehyde, aniline, and 2-hydroxynaphthalene-1,4-dione as a model.

covered catalyst was reused for at least four runs without any loss of activity (Fig. 2).

\section{Conclusions}

We have developed a green and simple protocol for the synthesis of 2-amino-3-cyano-4-aryl-5,10-dioxo-5,10-dihydro-4H-benzo $[g]$ chromenes and hydroxy-substituted naphthalene-1,4-dione derivatives via three-component condensation reactions using SBPPSA as a solid acid catalyst under ambient and solvent-free conditions. This procedure is a promising strategy and has several advantages such as clean reactions, easy workup, and eco-friendliness. It is expected that the present methodology will find application in organic synthesis.

\section{References}

[1] Zhu J, Bienaymé H. Multicomponent Reactions. Weinheim: WILEY-VCH Verlag, 2005

[2] Lakontseva E, Krasavin M. Tetrahedron Lett, 2010, 51: 4095

[3] Chrobok A, Baj S W, Pudło A. Appl Catal A, 2009, 366: 22

[4] Ough M, Lewis A, Bey E A, Gao J M, Ritchie J M, Bornmann W, Boothman D A, Oberley L W, Cullen J J. Cancer Biol Ther, 2005, 4: 95

[5] Moon D O, Choi Y H, Kim N D, Park Y M, Kim G Y. Int Immunopharmacol, 2007, 7: 506
[6] de Andrade-Neto V F, Goulart M O F, da Silva Filho J F, da Silva M J, Pinto M d C F R, Pinto A V, Zalis M G, Carvalho L H, Krettli A U. Bioorg Med Chem Lett, 2004, 14: 1145

[7] Pérez-Sacua E, Estérez-Braun A, Ravelo Á G, Yapu D G, Turba A G. Chem Biodivers, 2005, 2: 264

[8] Shestopalov A M, Emelianova Yu M, Nesterov V N. Russ Chem Bull, 2003, 52: 1164

[9] Hunger K. Industrial Dyes. Weinheim: WILEY-VCH Verlag, 2003

[10] Aldakov D, Anzenbacher P Jr. Chem Commun, 2003: 1394

[11] Gold H. In: Venkataraman H Ed. The Chemistry of Synthetic Dyes. New York: Academic Press, 1971

[12] Shaterian H R, Mohammadnia M.J Mol Liq, 2013, 177: 353

[13] Shaabani A, Ghadari R, Ghasemi S, Pedarpour M, Rezayan A H, Sarvary A, Ng S W.J Comb Chem, 2009, 11: 956

[14] Wang X H, Zhang X H, Tu S J, Shi F, Zou X, Yan S, Han Z G, Hao W J, Cao X D , Wua S S. J Heterocyclic Chem, 2009, 46: 832

[15] Yu Y, Guo H Y, Li X J. J Heterocyclic Chem, 2011, 48: 1264

[16] Yao C S, Yu C X, Li T J, Tu S J. Chin J Chem, 2009, 27: 1989

[17] Khurana J M, Nand B, Saluja P. Tetrahedron, 2010, 66: 5637

[18] Khodeir M, El-Taweel F, Elagamey A. Pharmazie, 1992, 47: 486

[19] Shaterian H R, Kangani M. Sci Iran, 2013, 20: 571

[20] Khurana J M, Magoo D, Chaudhary A. Synth Commun, 2012, 42: 3211

[21] Dabiri M, Tisseh Z N, Bazgir A. Dyes Pigment, 2011, 89: 63

[22] Shaterian H R, Moradi F. Res Chem Intermediate, DOI: 10.1007/ s11164-013-1191-3

[23] Shaterian H R, Khorami F, Doostmohammadi R, Amirzadeh A, Ghashang M. Phosphorus Sulfur Silicon, 2009, 184: 2227

[24] Shaterian H R, Doostmohammadi R, Khorami F, Ghashang M. Phosphorus Sulfur Silicon, 2010, 185: 171

[25] Shaterian H R, Khorami F, Amirzadeh A, Ghashang M. Phosphorus Sulfur Silicon, 2008, 183: 2865

[26] Shaterian H R, Amirzadeh A, Khorami F, Ghashang M. Synth Commun, 2008, 38: 2983

[27] Shaterian H R, Khorami F, Amirzadeh A, Ghashang M. Chin J Chem, 2009, 27: 815

[28] Niknam K, Deris A, Naeimi F, Majleci F. Tetrahedron Lett, 2011, 52: 4642

[29] Ghashang M, Mansoor S S, Aswin K. Res Chem Intermediate, DOI: 10.1007/s11164-013-1419-2

[30] Rahi T, Baghernejad M, Niknam K. Chin Chem Lett, 2012, 23 : 1103

[31] Niknam K, Tayebi S. Iran J Catal, 2012, 2(2): 69

\section{Graphical Abstract}

Chin. J. Catal., 2014, 35: 242-246 doi: 10.1016/S1872-2067(12)60761-X

\section{Silica-bonded propylpiperazine- $N$-sulfamic acid as recyclable solid acid catalyst for preparation of 2-amino-3-cyano-4-aryl- 5,10-dioxo-5,10-dihydro- $4 H$-benzo $[g]$ chromenes and hy- droxy-substituted naphthalene-1,4-dione derivatives}

Fahime Khorami, Hamid Reza Shaterian*

University of Sistan and Baluchestan, Iran

An efficient method for the synthesis of 2-amino-3-cyano-4-aryl-5,10dioxo-5,10-dihydro- $4 H$-benzo $[g]$ chromenes and hydroxy-substituted naphthalene-1,4-dione derivatives, using silica-bonded propylpiperazine- $N$-sulfamic acid (SBPPSA) as a solid acid, green, heterogeneous catalyst, under ambient and solvent-free conditions, is described.

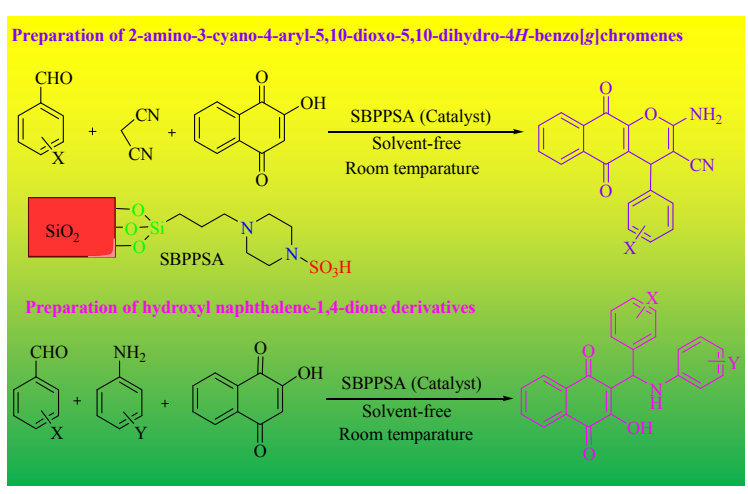

